PREPARED FOR THE U.S. DEPARTMENT OF ENERGY, UNDER CONTRACT DE-AC02-76CH03073

PPPL-3540

PPPL-3540

UC-70

Low Frequency Fishbone Mode Induced

by Circulating Particles in Spherical Tori

by

Ya.I. Kolesnichenko, V.S. Marchenko, and R.B. White

February 2001

MV|

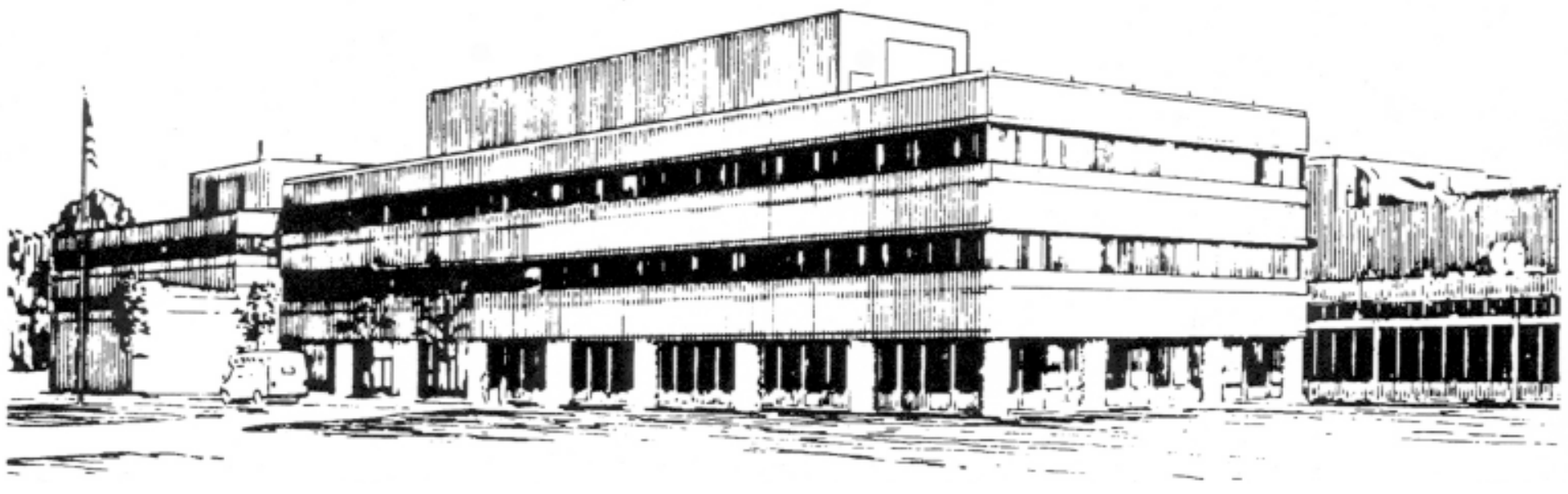

PRINCETON PLASMA PHYSICS LABORATORY PRINCETON UNIVERSITY, PRINCETON, NEW JERSEY 


\section{PPPL Reports Disclaimer}

This report was prepared as an account of work sponsored by an agency of the United States Government. Neither the United States Government nor any agency thereof, nor any of their employees, makes any warranty, express or implied, or assumes any legal liability or responsibility for the accuracy, completeness, or usefulness of any information, apparatus, product, or process disclosed, or represents that its use would not infringe privately owned rights. Reference herein to any specific commercial product, process, or service by trade name, trademark, manufacturer, or otherwise, does not necessarily constitute or imply its endorsement, recommendation, or favoring by the United States Government or any agency thereof. The views and opinions of authors expressed herein do not necessarily state or reflect those of the United States Government or any agency thereof.

\section{Availability}

This report is posted on the U.S. Department of Energy's Princeton Plasma Physics Laboratory Publications and Reports web site in Calendar Year 2001. The home page for PPPL Reports and Publications is: http://www.pppl.gov/pub_report/

DOE and DOE Contractors can obtain copies of this report from:

U.S. Department of Energy

Office of Scientific and Technical Information

DOE Technical Information Services (DTIS)

P.O. Box 62

Oak Ridge, TN 37831

Telephone: (865) 576-8401

Fax: (865) 576-5728

Email: reports@adonis.osti.gov

This report is available to the general public from:

National Technical Information Service

U.S. Department of Commerce

5285 Port Royal Road

Springfield, VA 22161

Telephone: 1-800-553-6847 or

(703) 605-6000

Fax: (703) 321-8547

Internet: http://www.ntis.gov/ordering.htm 


\title{
Low frequency fishbone mode induced by circulating particles in spherical tori
}

\author{
Ya.I. Kolesnichenko ${ }^{1}$, V.S. Marchenko ${ }^{1}$, R.B. White $^{2}$ \\ ${ }^{1}$ Scientific Centre "Institute for Nuclear Research", Kyiv, 03680, Ukraine \\ ${ }^{2}$ Princeton Plasma Physics Laboratory, P.O. Box 451, Princeton, New Jersey, \\ 08543, USA
}

(February 2, 2001)

\begin{abstract}
It is found that high $\beta$ in low-aspect-ratio tori tends to stabilize the fishbone instability in a plasma with energetic circulating ions. The stabilization results from enhancement of the toroidal drift motion by large Shafranov shift, which makes it difficult to reconcile the condition of considerable energy exchange between the ions and the internal kink perturbation with the condition of the resonant wave-particle interaction.
\end{abstract}

52.55.Hc, 52.35.Py, 52.50.Gj, 52.55.Pi

Typeset using REVTEX 
It is known that a considerable loss of the energetic ions in tokamaks can result from the fishbone instability $[1,2]$. Most of works on this topic deal with the instability associated with the trapped particles. However, experiments show that the fishbone mode can be also destabilized by the circulating particles, and a theory developed in Ref. [3] predicts that the growth rate of such an instability is of the same order as that of the trapped-particle-induced instability. On the other hand, experiments where circulating particles dominate in the energetic ion population indicate that the fishbone instability tends to be stabilized when $\beta$ (the ratio of the plasma pressure to the magnetic field pressure) is high [5], which was observed on Small Tight Aspect Ratio Tokamak (START) [4] Spherical Torus (ST). The stabilizing effect of high $\beta$ in STs was discovered theoretically in Ref. [6] (see also Ref. [7]) where, however, the trapped-particle-induced fishbone mode was considered. No attempt has been done yet to develop a theory relevant to the fishbone instability in a high- $\beta$ plasma with the circulating energetic ions. This issue is addressed in the present Letter.

There are two fishbone branches: the high frequency and low frequency ones $[8,9]$. The fishbone instability considered in Ref. [3] is relevant to the latter. We will also consider this branch assuming, as in Ref. [3], that the safety factor $q(r)$ is a monotonic function and that $m=n=1$ kink perturbation dominates, where $m$ and $n$ are the poloidal and toroidal mode number, respectively. However, in contrast to Ref. [3], we assume that the diamagnetic frequency of the bulk ions, $\omega_{* i}$, is much less than the frequency of the toroidal drift motion of the energetic ions, $\omega_{D}$, which implies that $\mathcal{E}_{b} / T \gg A^{2}$, where $\mathcal{E}$ is the particle energy, subscript "b" labels the beam ions, $T$ is the plasma temperature, $A$ is the aspect ratio of the torus (we used the estimate $\omega_{D} \sim \rho_{\|} v_{b} / R_{0}^{2}$ with $\rho_{\|}=\left|v_{\|}\right| / \Omega_{b}$ the "parallel Larmor radius", $\Omega$ the gyrofrequency, $v$ the particle velocity, and $R_{0}$ the radius of the magnetic axis). Note that the latter condition is well satisfied in STs and can be fulfilled even in conventional tokamaks. When it is satisfied, the circulating energetic ions interact with the mode through the resonance $k_{\|} v_{\|} \approx \omega_{D}$ (rather 
than through the resonance $\omega \approx k_{\|} v_{\|}$considered in Ref. [3]). Because $v_{\|} / R_{0} \gg \omega_{D}$ and $k_{\|} R_{0}=q^{-1}-1$, this resonance takes place only at a certain radius $\left(r_{*}\right)$ in the vicinity of the radius of the $q=1$ surface $\left(r_{s}\right)$, but not so close to $r_{s}$ as in the case of $\omega_{D} \ll \omega_{* i}$. Below we will show that $\omega_{D}$ grows with $\beta$, so that $\omega_{D}=\xi \rho_{\|} v_{b} / R_{0}^{2}$, where $\xi(\beta)>1$. Taking into account this fact and that $k_{\|} \approx s_{1}\left(\epsilon_{s}-\epsilon\right) / r_{s}$, where $s_{1}=s\left(r_{s}\right), s$ is the magnetic shear, $\epsilon_{s}=r_{s} / R_{0}$, we obtain $\left|r_{*}-r_{s}\right|=\xi_{s} \rho_{\|} \epsilon_{s} / s_{1}$ with $\xi_{s}=\xi\left(r_{s}\right)$. On the other hand, only particles crossing the $q=1$ surface can lead to strong instability (the energy exchange between other particles and the waves is small) [3]. Therefore, the instability arises provided that the resonance radius satisfies the condition $\left|r_{*}-r_{s}\right|<\Delta_{b}$, where $\Delta_{b}$ is the half-width of the orbits of the beam ions. In order to see whether it is satisfied in STs, we use $r_{*}$ found above and $\Delta_{b} \sim \rho_{\|}$. Then we obtain the condition $s_{1}>\xi_{s}(\beta) \epsilon_{s}$, which is difficult to satisfy in STs when $\beta$ is sufficiently large. Thus, we conclude that high $\beta$ and small aspect ratio of STs are the factors which tend to stabilize the fishbone instability associated with the circulating particles.

A more detailed analysis is required to find the magnitude of the reduction of the growth rate due to the described mechanism and the dependence of the stabilizing effect on the plasma pressure, which will be carried out below. First of all, we obtain an expression for the frequency of the toroidal drift motion of the well circulating particles in an equilibrium plasma. We follow the canonical description of the orbits introduced in Ref. [10]. Using the frame of reference where the equilibrium electric field is absent, we write:

$$
\dot{\theta}=\frac{v_{\|}}{q R}-\frac{c m v_{\|}^{2}}{e R} \frac{\partial R}{\partial \psi}
$$

where $R$ is the distance from the major axis of the torus, $\psi$ is the toroidal flux, $\theta$ is the poloidal coordinate related to the corresponding Shafranov coordinate (labeled by $S$ ) in accordance with the expression [10]

$$
R=R_{0}-\Delta+r \cos \theta_{S}=R_{0}-\Delta+r \cos \theta+\eta r(\cos 2 \theta-1)+R_{0} O\left(\epsilon^{3}\right)
$$


with $\eta(r)=0.5\left(\Delta^{\prime}+r / R_{0}\right) \sim \epsilon, \Delta^{\prime}=d \Delta / d r$, and $\Delta(r)>0$ is the Shafranov shift, $\Delta(0)=0$. Combining Eqs. (2), (1) and carrying out the orbit averaging, we obtain:

$$
\langle\dot{\theta}\rangle=\frac{v_{\|}}{q R_{0}}+\omega_{D}
$$

where

$$
\omega_{D}=\frac{\rho_{\|}\left|v_{\|}\right|}{2 r R_{0}}\left(2 \epsilon+3 \Delta^{\prime}+r \Delta^{\prime \prime}\right)
$$

the radial coordinate $r$ is defined by $\psi(r)=\int^{r} d r r B_{0}, B_{0}$ is the magnetic field at the magnetic axis. When deriving this equation, it was assumed that the orbit width is small compared to the shear length. Theory of the tokamak equilibrium provides an ordinary differential equation for $\Delta$. Corresponding expressions for $\Delta^{\prime}$ and $r \Delta^{\prime \prime}$ are well known [11]:

$$
\begin{gathered}
\Delta^{\prime}=\epsilon\left(\beta_{\theta}+0.5 l_{i}\right), \\
r \Delta^{\prime \prime}=\epsilon\left[1-(3-2 s)\left(\beta_{\theta}+0.5 l_{i}\right)\right]+\alpha_{p},
\end{gathered}
$$

where $l_{i}=2 /\left(r^{2} B_{\theta}^{2}\right) \int_{0}^{r} B_{\theta}^{2} r d r$ is the internal inductance per unit length, $\beta_{\theta}=$ $\left(8 \pi / B_{\theta}^{2}\right)(\bar{p}-p)$ with $\bar{p}=\left(2 / r^{2}\right) \int_{0}^{r} p r d r$ the average pressure, and $\alpha_{p}=$ $-\left(8 \pi p^{\prime} / B_{0}^{2}\right) R_{0} q^{2}$. Substituting Eqs (5), (6) into Eq. (4) we find that $\omega_{D}=$ $\xi \rho_{\|}\left|v_{\|}\right| /\left(R_{0}^{2}\right)$, where

$$
\xi=\frac{3}{2}+s\left(\beta_{\theta}+\frac{l_{i}}{2}\right)+\frac{\alpha_{p}}{2 \epsilon} .
$$

It follows from Eq. (7) that the Shafranov shift strongly increases the frequency of the toroidal drift motion in STs with high- $\beta$ plasmas (for parabolic profiles $\xi \sim 3$ ).

However, Eq. (7) was obtained for a plasma with the circular cross section. To find $\omega_{D}$ in a plasma with the arbitrary shape of the cross section, we use the magnetic coordinates $[12,13]$ and take the magnetic field strength in the form $[6,7]$ :

$$
B=B_{0}\left[1-\epsilon \cos \theta+\epsilon^{2}\left(\alpha+\sigma \cos ^{2} \theta\right)\right]
$$


where $\alpha$ and $\sigma$ are adjustable parameters, $\alpha>>1, \sigma \gtrsim 1$ in STs with high $\beta$. Taking into account that the drift velocity is $\mathbf{v}_{D} \approx v_{\|}^{2} \mathbf{b} \times \mathcal{K} / \Omega$, where $\mathcal{K}$ is the field line curvature, we write:

$$
\omega_{D}=-\frac{\omega_{b}}{\Omega_{0}} \oint \frac{d \theta}{2 \pi} \frac{q\left|v_{\|}\right| B_{3}}{r}\left(\frac{\partial}{\partial r} \frac{1}{B}-\frac{4 \pi}{|B|^{3}} \frac{\partial p}{\partial r}\right),
$$

where $\omega_{b}$ is the transit time frequency, $\Omega_{0}=e B_{0} /(M c), B_{3}=B_{3}(r)$ is a covariant component of B. Eqs. (8), (9) yield:

$$
\xi=1+2 \alpha+\sigma-\frac{\alpha_{p}}{2 \epsilon q^{2}}
$$

Typically, $\alpha_{p} /\left(2 \epsilon q^{2}\right) \sim 1$ or less. Therefore, $\xi \gg 1$ in high- $\beta$ plasmas with any shape of the flux surfaces.

A general expression for the resonance describing the interaction of the wellcirculating particles and the $m=n=1$ perturbation is given by (the time and angle dependence of the perturbation is taken in the form $\exp (-i \omega t-i \phi+i \theta))$ :

$$
\omega-\left(k_{\|}+\frac{S}{q R}\right) v_{\|}-\omega_{D}=0
$$

where $S$ is an integer, $k_{\|}=\left(q^{-1}-1\right) / R_{0}$. Because $v /\left(q R_{0}\right)$ well exceeds both $\omega$ and $\omega_{D}$, we have to take $S=0$. With this resonance condition, the kinetic part of the potential energy $\delta W_{k}$ is given by [3]:

$$
\begin{gathered}
\delta W_{k}=-\frac{\pi}{\left(B_{\theta} s\right)_{r_{s}}^{2}} \int_{0}^{r_{s}} r h(r) d r \\
h(r)=-\frac{8}{\pi} \frac{1}{R_{0}^{2}} \int d \mathbf{v} \mathcal{E}^{2} \frac{\partial F_{b}}{\partial P_{\phi}} \frac{\Delta_{b}}{\left|\Delta_{b}\right|} \int_{-\pi}^{\pi} \cos \theta \frac{H(1-|z|) \sqrt{1-z^{2}}}{\omega-k_{\|}\left(r_{s}+z \Delta_{b}\right) v_{\|}-\omega_{D}\left(r_{s}\right)} d \theta,
\end{gathered}
$$

where $z=\left[\bar{r}(r, \theta)-r_{s}\right] / \Delta_{b}, \bar{r}=r+\Delta_{b} \cos \theta, P_{\phi}=M v_{\|} R+e \psi / c$ is the canonical angular momentum, $H(x)$ is the Heaviside unit step function. As $|z|<1$ and $\Delta_{b} \ll r_{s}$, the resonance condition (which is determined by the denominator of Eq. (13)) can be written as follows:

$$
\omega+s_{1} \frac{v_{\|}^{2} z}{\Omega_{b} R_{0} r_{s}}-\frac{\xi_{s} v_{\|}^{2}}{\Omega_{b} R_{0}^{2}}=0 .
$$


We assume that beam ions are characterized by the vanishing magnetic moment, $\mu$, and take their distribution function in the following form:

$$
F_{b}=\frac{\sqrt{2} m_{b}^{3 / 2}}{\pi \mathcal{E}_{\alpha}} p_{b}(r) H\left(\mathcal{E}_{\alpha}-\mathcal{E}\right) \frac{\delta\left(\mu B_{0} / \mathcal{E}\right)}{\mathcal{E}_{c}^{3 / 2}+\mathcal{E}^{3 / 2}}\left[\frac{1}{2}(1-\zeta)+\zeta H\left( \pm v_{\|}\right)\right]
$$

where $p_{b}(r)$ is the beam ion pressure, $\mathcal{E}_{\alpha}$ the injection energy, $\mathcal{E}_{c} \sim\left(m_{i} / m_{e}\right)^{1 / 3} T$ the energy for which the ions and electrons equally contribute to the slowing down of the beam particles, $\zeta=0$ for the balanced injection, and $\zeta=1$ for the co- and counterinjection. Combining (12)-(14), inverting the order of integration $\int d r \int d \theta \int d \mathbf{v}=$ $\int d \mathbf{v} \int d \theta \int d r$ with $d r \simeq \Delta_{b} d z$, and assuming $\operatorname{Re} \omega \gg \operatorname{Im} \omega$, we obtain:

$$
\operatorname{Im} \delta W_{k}=-\epsilon_{s}\left[\frac{\Delta_{b}^{\alpha}}{r_{p b}} \frac{\beta_{b \theta}}{s^{3}}\right]_{r_{s}} I
$$

where

$$
I \equiv \int_{0}^{1} \sqrt{x}\left[1-\left(\frac{\mathcal{E}_{s}}{\mathcal{E}_{\alpha} x}-\kappa\right)^{2}\right] H\left(1-\left|\frac{\mathcal{E}_{s}}{\mathcal{E}_{\alpha} x}-\kappa\right|\right) d x
$$

$\kappa \equiv \xi_{s} \epsilon_{s} / s_{1}, \beta_{b \theta}$ is the poloidal beta of beam ions, $\mathcal{E}_{s} \equiv m_{b} \Omega_{b} \omega R_{0} r_{s} /\left(2 s_{1}\right)$, and $r_{p b}^{-1}=-d \ln p_{b} / d r$.

One can see that when $\xi_{s} \epsilon_{s} \ll s_{1}(\kappa \ll 1)$, the integral $I$ and, thus, the instability growth rate are maximum. In this case Eqs. (16) (17) give the result by Betti and Freidberg (BF) [3]:

$$
\operatorname{Im} \delta W_{k}^{B F} \approx-\left.\frac{2 \epsilon_{s} \Delta_{b}^{\alpha} \beta_{b \theta}}{3 s^{3} r_{p b}}\right|_{r_{s}}
$$

Let us consider the case of $\kappa>1+\mathcal{E}_{s} / \mathcal{E}_{\alpha}$, which is of the main interest for STs. We obtain:

$$
\begin{array}{r}
I=2\left(\frac{\mathcal{E}_{s}}{\mathcal{E}_{\alpha}}\right)^{3 / 2}\left\{\frac{2 \kappa}{\sqrt{\kappa-1}}-\frac{2 \kappa}{\sqrt{\kappa+1}}\right. \\
\left.+\frac{1}{3}\left(1-\kappa^{2}\right)\left[(\kappa-1)^{-3 / 2}-(\kappa+1)^{-3 / 2}\right]+\sqrt{\kappa-1}-\sqrt{\kappa+1}\right\} .
\end{array}
$$

We observe that $I$ can be much less than $I^{B F}$ for realistic magnitudes of $\kappa$ in STs: $I / I^{B F} \sim\left(\mathcal{E}_{s} / \mathcal{E}_{\alpha}\right)^{1.5} \sim\left(T R_{0}\right)^{1.5} /\left(\mathcal{E}_{\alpha} s_{1} r_{p i}\right)^{1.5}$ for $\kappa \gtrsim 1$ and $\omega=\omega_{* i}$, where $r_{p i}^{-1}=-d \ln p_{i} / d r$. But it vanishes only for $\kappa \rightarrow \infty$. The reason why $\operatorname{Im} \delta W_{k}$ 
does not vanish for the finite $\kappa$ is the presence of particles with $\mathcal{E} \ll \mathcal{E}_{\alpha}$ for which $\left|r_{*}-r_{s}\right|<\Delta_{b}$ due to the resonance $\omega \approx k_{\|} v_{\|}$.

A simple estimate shows that when $\mathcal{E}_{s} / \mathcal{E}_{\alpha} \simeq 0.1, \kappa=1.2$ is sufficient for more than the tenfold decrease of the BF response. Using Eq. (7) we find that the condition $\kappa \geq 1.2$ yields the following restriction on $\beta$ for the plasma with the parabolic profile of the pressure (in which case $\alpha_{p}=2 \epsilon_{s} \beta(0) A^{2}, \beta_{\theta}\left(r_{s}\right)=\beta(0) A^{2} / 2$ with $\beta(0)=2 \bar{\beta}, \bar{\beta}$ is the volume averaged $\beta)$ :

$$
\bar{\beta} \geq \frac{1}{\left(2+s_{1}\right) A^{2}}\left(1.2 \frac{s_{1}}{\epsilon_{s}}-\frac{3}{2}-\frac{s_{1} l_{i}}{2}\right) .
$$

When this condition is satisfied, the amplitude of fishbones is small or the instability is completely stabilized due to the presence of weak damping mechanisms.

It is of interest to see whether Eq. (20) is satisfied in experiments on START where fishbone oscillations were weak or disappeared in high- $\beta$ discharges with $\langle\beta\rangle \sim 30 \%$ ) [5]. To make an estimate we take $A=1.5, \mathcal{E}_{\alpha}=30 \mathrm{keV}$, $T=300 \mathrm{eV}, l_{i}=0.7, s_{1} \sim 0.5$, and $r_{s} \sim a / 3$. Then we can write Eq. (20) as $\bar{\beta} \geq 20 \%$. For the used parameters $\mathcal{E}_{s} / \mathcal{E}_{\alpha} \sim 0.03$, which leads to $I / I_{B F} \sim 10^{-2}$. This result together with the prediction of the complete stabilization of the trappedparticle-induced fishbone mode at high $\beta[6,7]$ may explain the disappearance of fishbones in START.

In conclusion, we considered for the first time the stability of the fishbone mode associated with the circulating ions in high- $\beta$ plasmas of spherical tori. We have shown that well-circulating energetic ions undergo strong toroidal drift motion when the aspect ratio of the torus is small and the plasma pressure is high. Because of this enhanced drift motion, the radius $r_{*}$ (where the resonance between the energetic ions crossing the $q=1$ surface and the internal kink perturbation occurs) may be shifted for the distance exceeding the particle orbit width, which stabilizes the instability. This conclusion together with the prediction of complete stabilization of the fishbones associated with the energetic trapped ions $[6,7]$ indicate that STs with high $\beta$ have the immunity to the fishbone instability. In order to draw a 
final conclusion on the fishbone oscillations in STs, experimental verification of the theoretical predictions is required (in particular, this can be done in current NBI experiments on the National Spherical Torus Experiment, NSTX [14]). In addition, a theory of the high-frequency fishbone branch in STs with the tangential injection should be developed.

The research described in this publication was made possible in part by Award No. UP2-2114 of the Government of Ukraine and CRDF, U.S. Department of Energy Grant DE-AC02-76CH03073, and was also supported by the IAEA contract No. 10539. The authors thank Yu. Yakovenko for useful discussions. 


\section{REFERENCES}

[1] K. McGuire, R. Goldston, M. Bell, et al., Phys. Rev. Lett. 50, 891 (1983).

[2] W.W. Heidbrink, K. Bol, D. Buchenauer, et al., Phys. Rev. Lett. 57, 835 (1986).

[3] R. Betti, J.P. Freidberg, Phys. Rev. Lett. 70, 3428 (1993).

[4] A. Sykes et al., Nucl. Fusion 32, 694 (1992).

[5] R. Akers, et al., Plasma Phys. Control. Fusion (Proc. 26 EPS Conf., Maastricht, 1999) 23J, 117 (1999).

[6] Ya.I. Kolesnichenko, V.V. Lutsenko, and V.S. Marchenko, Phys. Rev. Lett. 82, 3260 (1999).

[7] Ya.I. Kolesnichenko, V.V. Lutsenko, and V.S. Marchenko, Nucl. Fusion 40, $1731(2000)$.

[8] L. Chen, R.B. White, and M.N. Rosenbluth, Phys. Rev. Lett. 52, 1122 (1984).

[9] B. Coppi and F. Porcelli, Phys. Rev. Lett. 57, 2272 (1986).

[10] J.D. Meiss and R.D. Hazeltine, Phys. Fluids B 2, 2563 (1990).

[11] R.B. White, Theory of Tokamak Plasmas, North-Holland, Amsterdam, (1989).

[12] A.H. Boozer, Phys. Fluids 24, 1999 (1981).

[13] R.B. White and M.S. Chance, Phys. Fluids 27, 2455 (1984).

[14] J. Spitzer, et al., Fusion Technol. 30, 1337 (1996). 


\section{External Distribution}

Plasma Research Laboratory, Australian National University, Australia

Professor I.R. J ones, Flinders University, Australia

Professor J oão Canalle, Instituto de Fisica DEQ/IF - UERJ , Brazil

Mr. Gerson O. Ludwig, Instituto Nacional de Pesquisas, Brazil

Dr. P.H. Sakanaka, Instituto Fisica, Brazil

The Librarian, Culham Laboratory, England

Library, R61, Rutherford Appleton Laboratory, England

Mrs. S.A. Hutchinson, JET Library, England

Professor M.N. Bussac, Ecole Polytechnique, France

Librarian, Max-Planck-Institut für Plasmaphysik, Germany

J olan Moldvai, Reports Library, MTA KFKI-ATKI, Hungary

Dr. P. Kaw, Institute for Plasma Research, India

Ms. P.J . Pathak, Librarian, Insitute for Plasma Research, India

Ms. Clelia De Palo, Associazione EURATOM-ENEA, I taly

Dr. G. Grosso, Instituto di Fisica del Plasma, Italy

Librarian, Naka Fusion Research Establishment, J AERI, J apan

Library, Plasma Physics Laboratory, Kyoto University, J apan

Research Information Center, National Institute for Fusion Science, J apan

Dr. O. Mitarai, Kyushu Tokai University, J apan

Library, Academia Sinica, Institute of Plasma Physics, People's Republic of China

Shih-Tung Tsai, Institute of Physics, Chinese Academy of Sciences, People's Republic of China

Dr. S. Mirnov, Triniti, Troitsk, Russian Federation, Russia

Dr. V.S. Strelkov, Kurchatov Institute, Russian Federation, Russia

Professor Peter Lukac, Katedra Fyziky Plazmy MFF UK, Mlynska dolina F-2, Komenskeho Univerzita, SK-842 15 Bratislava, Slovakia

Dr. G.S. Lee, Korea Basic Science Institute, South Korea

Mr. Dennis Bruggink, Fusion Library, University of Wisconsin, USA

Institute for Plasma Research, University of Maryland, USA

Librarian, Fusion Energy Division, Oak Ridge National Laboratory, USA

Librarian, Institute of Fusion Studies, University of Texas, USA

Librarian, Magnetic Fusion Program, Lawrence Livermore National Laboratory, USA

Library, General Atomics, USA

Plasma Physics Group, Fusion Energy Research Program, University of California at San Diego, USA

Plasma Physics Library, Columbia University, USA

Alkesh Punjabi, Center for Fusion Research and Training, Hampton University, USA

Dr. W.M. Stacey, Fusion Research Center, Georgia Institute of Technology, USA

Dr. J ohn Willis, U.S. Department of Energy, Office of Fusion Energy Sciences, USA

Mr. Paul H. Wright, Indianapolis, Indiana, USA 
The Princeton Plasma Physics Laboratory is operated by Princeton University under contract with the U.S. Department of Energy.

\author{
Information Services \\ Princeton Plasma Physics Laboratory \\ P.O. Box 451 \\ Princeton, NJ 08543
}

Phone: 609-243-2750

Fax: 609-243-2751

e-mail: pppl_info@pppl.gov

Internet Address: http://www.pppl.gov 